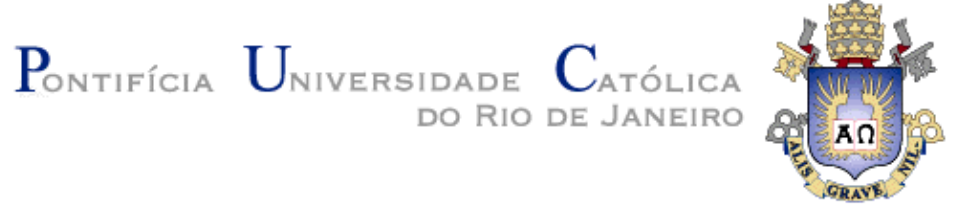

Fernando Antonio Lucena Aiube

Modelagem dos Preços Futuros de Commodities:

Abordagem pelo Filtro de Partículas

Tese de Doutorado

Tese apresentada ao Programa de Pós-graduação em Engenharia Produção do Departamento de Engenharia Industrial da PUC-Rio como parte dos requisitos parciais para obtenção do título de Doutor em Engenharia de Produção.

Orientador: Tara Keshar Nanda Baidya

Rio de Janeiro

Julho de 2005 


\section{Modelagem dos Preços Futuros de Commodities: Abordagem pelo Filtro de Partículas}

Tese apresentada como requisito parcial para obtenção do título de Doutor pelo Programa de Pós-Graduação em Engenharia de Produção da PUC-Rio. Aprovada pela Comissão Examinadora abaixo assinada.

Prof. Tara Keshar Nanda Baidya Orientador Departamento de Engenharia Industrial - PUC - Rio

Prof. Reinaldo Castro de Souza Departamento de Engenharia Elétrica - PUC - Rio

Edison Américo Huarsaya Tito PUC-Rio

Profa. Mônica Barros Departamento de Engenharia Elétrica - PUC - Rio

Prof. Carlos Patrício Samanez Departamento de Engenharia Industrial - PUC - Rio

Profa. Telma Sáfadi Departamento de Ciências Exatas - Universidade Federal de Lavras

Prof. Love Ekenberg Department of Computer and Systems Sciences - University of Stockholm

Prof. José Eugênio Leal Coordenador(a) Setorial do Centro Técnico Científico - PUC-Rio 
Todos os direitos reservados. É proibida a reprodução total ou parcial do trabalho sem autorização da universidade, do autor e do orientador.

\section{Fernando Antonio Lucena Aiube}

Graduou-se em Engenharia Elétrica pela Universidade Federal de Goiás em 1980. Especializou-se em Engenharia de Petróleo na Petrobras em 1981. Exerceu a atividade de engenheiro de petróleo na área de avaliação de poços e análise econômica de projetos petrolíferos. Obteve o título de Mestre em Engenharia de Produção na Pontifícia Universidade Católica do Rio de Janeiro em 1995, tendo como concentração a área de Finanças e Análise de Investimentos e linha de pesquisa a Teoria das Opções Reais. Trabalha atualmente no Planejamento Financeiro e Análise de Portfólio da Petrobras e leciona no curso de graduação do Departamento de Engenharia Industrial da PUC-Rio.

Ficha catalográfica

Aiube, Fernando Antonio Lucena
Modelagem dos preços futuros de commodities :
abordagem pelo filtro de partículas / Fernando Antonio
Lucena Aiube ; orientador: Tara Keshar Nanda Baidya. -
Rio de Janeiro : PUC-Rio, Departamento de Engenharia
Industrial, 2005.
$\quad 183$ f. ; $30 \mathrm{~cm}$
Tese (doutorado) - Pontifícia Universidade Católica do
Rio de Janeiro, Departamento de Engenharia Industrial
Inclui referências bibliográficas
1. Engenharia Industrial - Teses. 2. Processos
estocásticos. 3. Filtro de Kalman. 4. Filtro de partículas. 5.
Opções reais. 6. Validação cruzada. I. Souza, Reinaldo
Castro. II. Pontifícia Universidade Católica do Rio de
Janeiro. Departamento de Engenharia Industrial . III. Título.

CDD: 658.5 


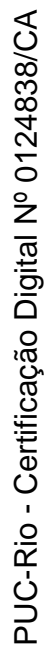

Ao meu irmão Carlos de Lucena Aiube 


\section{Agradecimentos}

Ao meu orientador Professor Tara Baidya pelo incentivo para iniciar esta jornada e pela paciência e dedicação na condução da orientação.

Ao colega da Petrobras/PUC Edison Tito pela orientação na metodologia do filtro de partículas.

Aos professores Reinaldo Castro de Souza e Cristiano Fernandes do Departamento de Engenharia Elétrica, pelas críticas apresentadas ao longo do desenvolvimento da pesquisa.

À PUC-Rio pelo auxílio através de bolsa integral nos últimos dezoito meses.

À Petrobras pelo tempo concedido para os estudos. 


\section{Resumo}

Aiube, Fernando Antonio Lucena. Modelagem dos Preços Futuros de Commodities: Abordagem pelo Filtro de Partículas. Rio, 2005. 183p. Tese de Doutorado - Departamento de Engenharia Industrial, Pontifícia Universidade Católica do Rio de Janeiro.

A evolução dos conhecimentos em Finanças nas últimas três décadas foi rápido e vertiginoso. Hoje os mercados financeiros oferecem produtos sofisticados para investidores e empresas, e por outro lado, tais agentes demandam instrumentos confiáveis para atender suas necessidades em busca de maiores retornos e menores riscos. Todo esse desenvolvimento baseia-se fundamentalmente em metodologias de apreçamento de ativos. Grande parte deste conhecimento é oriundo dos trabalhos pioneiros de Black e Scholes (1973) e Merton (1973). Em síntese, estes trabalhos apoiaram-se em processos estocásticos para preços de ativos para apreçar um derivativo. A natureza do processo estocástico de evolução dos preços é o ponto central para a derivação dos modelos de apreçamento. A análise do comportamento dos preços das commodities possui duas grandes vertentes na literatura. A primeira trata os preços como decorrência de modelos de equilíbrio entre a oferta e a demanda. Estes modelos prosperaram pouco em termos de pesquisa. A outra vertente trata da análise da evolução dos preços baseando-se na série histórica propriamente dita. Esta linha de pesquisa está mais presente na literatura. Esta tese concentra-se nesta abordagem. As commodities possuem características particulares principalmente porque a formação de preços ocorre, via de regra, em mercados futuros. Isto faz com que muitos fatos estilizados não possam ser descritos por modelos de um fator (ou uma variável estocástica). Os fatores (variáveis estocásticas) ou variáveis de estado em muitas situações não são observáveis e necessitam ser estimados. Os modelos de preços futuros, escritos como função das variáveis de estado, recebe o nome de equação de observação. Quando as variáveis de estado são Gaussianas e a equação de observação é linear nos estados, o problema pode ser estimado pelo filtro de Kalman clássico. Se ocorrer a não linearidade, esta dificuldade pode ser contornada pelo filtro de Kalman estendido. Quando o problema é não Gaussiano a literatura usa outras metodologias (freqüentemente aproximações) que não o filtro de Kalman. Esta tese trata de processos estocásticos para preços de 
commodities propondo extensões aos modelos existentes na literatura. A derivação dos modelos é feita com o uso da transformada de Duffie e Kan (1996) em ambiente de não arbitragem. Algumas das extensões incluem modelos não Gaussianos. Esta tese investiga a estimação destes modelos pela metodologia denominada filtro de partículas. O filtro de partículas é um procedimento recursivo para integração, dentro da classe dos métodos seqüenciais de MonteCarlo. A proposta de utilização desta metodologia decorre do fato de que ela dispensa as condições de linearidade e Gaussianidade. Dentre as contribuições desta tese destacam-se as extensões dos processos estocásticos aplicáveis para quaisquer commodities e as análises de modelos não Gaussianos através da metodologia do filtro de partículas. Além disso, a pesquisa apresenta: (i) conclusões acerca dos modelos de dois fatores aplicados à série de preços da commodity petróleo; (ii) a análise da viabilidade do filtro de partículas mostrando que o erro obtido é próximo daquele do filtro de Kalman para problemas Gaussianos e a resposta obtida da estimação paramétrica é coerente com diversos trabalhos da literatura; (iii) análise da viabilidade operacional de implementação do filtro de partículas em termos do tempo computacional despendido nos processos de filtragem e estimação paramétrica. A tese conclui que o filtro de partículas, apesar ser computacionalmente intenso, é viável na prática face ao imenso desenvolvimento computacional. Ainda mais, por ser uma metodologia aplicável a problemas complexos de inferência, sua utilização em modelos cada vez mais sofisticados é muito promissora.

\section{Palavras-chave}

Processos estocásticos; Filtro de Kalman; Filtro de Partículas; Opções Reais. 


\section{Abstract}

Aiube, Fernando Antonio Lucena. Modelling Commodity Future Prices: Particle Filter Approach. Rio, 2005. 183p. Ph.D. Thesis - Department of Industrial Engineering, Pontifical Catholic University of Rio de Janeiro.

The evolution of the ideas in Finance has been huge in the last decades. Nowadays the financial markets offer investors sophisticated products. And investors in turn demand reliable financial instruments to meet their needs in search for greater returns and lower risks. This development is based mainly on asset pricing methodologies. The greatest part of this knowledge comes from the seminal works of Black and Scholes (1973) and Merton (1973). To summarize, their works are based on the assumption of a specific stochastic process that governs asset prices. And then a derivative of this underlying asset can be priced. The nature of the stochastic process that describes the evolution of prices is the key point for deriving pricing formulae. The analysis of the behavior of commodity prices has two approaches. The first approach considers prices as a consequence of the equilibrium between supply and demand. These models have not received enough attention in literature. The second approach, which has received more attention, is based on the analysis of price time series. The commodities have particular features because they are most of the times negotiated in future markets. The consequence is that the one factor models badly describe their stylized facts. The factors (stochastic variables) are known as state variables which most of the times are non observables, and need to be estimated. When state variables are Gaussians and the observation equation is linear in states, the classical Kalman filter can be used to access these variables. If non linearity is present extended Kalman filter is used, but when state variables are non Gaussian the literature does not use filtering processes. This thesis analyses the stochastic processes of commodities proposing extensions to the existing models. The derivation of models is based on Duffie and Kan (1996) transform, in a non arbitrage environment. Some extensions are non Gaussian. This thesis investigates the estimation of these models using particle filter methodology. The particle filter is a recursive procedure for integration in the sequential Monte-Carlo methods. The advantage of this methodology is that it does not require linear or Gaussian 
conditions. The contributions of this research are the extensions of stochastic processes that can be used for any commodity and the use of particle filter as an estimation methodology in Finance. Furthermore the thesis presents: (i) the conclusions about two factor models applied to oil prices; (ii) the analysis of the use of particle filter verifying that errors in both, Kalman filter and particle filter are close and that parameters estimation is in accordance with the literature; (iii) the analysis of the implementation of particle filter showing that it is viable considering the computational time of filtering and parameters estimation. The thesis concludes that the particle filter is viable, although time consuming, due to the hardware development. And more, since particle filter is useful for complex inference problems, its application to sophisticated models is promising.

\section{Keywords}

Stochastic processes; Kalman Filter; Particle Filter; Real Options. 


\section{Sumário}

1 Introdução 19

1.1. O Contexto da Pesquisa 19

1.2. Organização da pesquisa 25

1.3. Mercados futuros - conceitos básicos 26

1.3.1. Conceitos básicos 26

1.3.2. Preços futuros - economia sem incerteza 28

1.3.3. Futuros de commodities $\quad 30$

1.3.4. Futuros de commodities - economia com incerteza 31

2 Revisão bibliográfica 35

2.1. Artigos diretamente relacionados à pesquisa 36

2.2. Artigos indiretamente relacionados com a pesquisa 46

3 Derivação dos modelos $\quad 52$

3.1. Introdução 53

3.2. Aspectos preliminares das transformadas DK e DPS 54

3.3. Formulação das transformadas DK e DPS 56

3.4. O conceito da transformada DK 57

3.5. O conceito da transformada estendida DPS 58

3.6. Derivação dos modelos com a transformada DK 59

3.6.1. Modelo com distribuição normal para os saltos e MGB para 61

3.6.2. Modelo com distribuição exponencial para os saltos e MGB para §63

3.6.3. Modelo com distribuição normal para os saltos e reversão à média para $\xi \quad 65$

3.7. Resumo dos modelos 66

$\begin{array}{ll}\text { 3.7.1. Modelo Básico } & 67\end{array}$

3.7.2. Modelo Primeira Extensão 69

3.7.3. Modelo Segunda Extensão 70

3.7.4. Modelo Terceira Extensão 72

4 Filtro de Kalman $\quad 74$

$\begin{array}{ll}\text { 4.1. Introdução } & 74\end{array}$ 
4.2. Definição do modelo na forma espaço-estado

4.3. As origens computacionais do filtro de Kalman 76

4.4. As origens probabilísticas do filtro 77

4.5. O algoritmo do filtro de Kalman 77

4.6. Estimação por máxima verossimilhança 79

4.7. Previsão 81

4.8. Alisamento 81

4.9. O filtro de Kalman estendido 82

5 Filtro de partículas $\quad 85$

5.1. Introdução 85

5.2. Definições básicas $\quad 87$

5.3. Amostragem por importância 88

5.4. Amostragem por importância seqüencial 90

5.5. Estratégia de reamostragem e o filtro bootstrap 91

6 Análise do processo de filtragem 96

6.1. Os filtros de Kalman e de partículas 96

6.2. Análise com dados artificiais 98

6.3. Análise com dados de mercado 108

6.4. Tamanho efetivo da amostra e a eficiência do FP 109

7 Análise dos resultados empíricos 111

7.1. Organização dos dados 112

7.2. Utilização dos painéis $\quad 114$

7.3. Sazonalidade 114

7.4. Estimação dos parâmetros 115

7.5. Resultados do modelo básico 117

7.5.1. Filtro de Kalman 118

7.5.2. Filtro de partículas 122

7.5.3. Tamanho efetivo da amostra no FP 127

7.5.4. Estrutura a termo da volatilidade 128

7.5.5. Estrutura a termo dos preços 132

7.5.6. Previsão 135

7.6. Resultados do Modelo Primeira Extensão 136

7.6.1. Filtro de Kalman 137 
7.6.2. Filtro de partículas

7.7. Resultados do Modelo Segunda Extensão 143

7.7.1. Modelos com distribuição normal para os saltos 143

7.7.2. Modelos com distribuição exponencial para os saltos 148

7.8. Resultados do Modelo Terceira Extensão 152

8 Aplicações e exemplos $\quad 155$

8.1. Implicações para a área de Opções Reais 155

8.2. Implicações para a área de Finanças 158

9 Conclusões e trabalhos futuros 163

10 Referências Bibliográficas 168

11 Apêndice 1 - Solução das EDOs com distribuição normal 175

12 Apêndice 2 - Solução das EDOs com distribuição exponencial 177

13 Apêndice 3 - Solução das EDOs com reversão à média para $\xi 179$

14 Apêndice 4 - Estrutura a termo da volatilidade - Modelo Básico 181

15 Apêndice 5 - Estrutura a termo da volatilidade - o Modelo Primeira

Extensão 


\section{Lista de figuras}

Figura 1 - Uma das possíveis trajetórias de preços gerada artificialmente 101

Figura 2 - Variável de observação utilizada nos filtros de Kalman e de partículas

Figura 3 - Variável de observação e a variável de observação pelo FK 102

Figura 4 - Variável de observação e a variável de observação filtrada pelo FP 103

Figura 5 - Erros (RMSE) da simulação com 100 experimentos usando o FP 105

Figura 6 - Erros (RMSE) para o caso de 100 experimentos usando 1000 partículas

106

Figura 7 - Comportamento assintótico do erro no FP em função do número de partículas

107

Figura 8 - Variável de estado (variações de curto prazo) do painel A 124

Figura 9 - Variável de estado (preços de equilíbrio) do painel A 124

Figura 10 - Evolução dos preços à vista estimado e do primeiro contrato futuro

Figura 11 - Estrutura a termo da volatilidade do painel A - FK

Figura 12 - Estrutura a termo da volatilidade do painel A - FP

Figura 13 - Estrutura a termo da volatilidade do painel C - FK

Figura 14 - Estrutura a termo da volatilidade do painel C - FP

Figura 15 - Preços futuros: variáveis observada e filtrada para o F9 - FK

Figura 16 - Preços futuros: variáveis observada e filtrada para o F9 - FP

Figura 17 - Previsão do contrato F1 de 21/09/00 a 12/12/01

Figura 18 - Posição nos contratos futuros de um mês e um ano 


\section{Lista de tabelas}

Tabela 1 - Erros na variável de observação com as duas metodologias de filtragem

Tabela 2 - Erros na variável de observação usando 1000 partículas no FP 105

Tabela 3 - Erro RMSE e tempo em função do número de partículas 106

Tabela 4 - Erro na variável de estado S com as duas metodologias $\quad 107$

Tabela 5 - Erros na variável de observação - preços do contrato F1 108

Tabela 6 - Variação do erro da variável de observação em função do valor de coef

Tabela 7 - Principais informações dos painéis utilizados 113

Tabela 8 - Utilização dos painéis em cada modelo 114

Tabela 9 - Resultados da estimação com o painel A pelo FK 119

Tabela 10 - Resultados da estimação com o painel C pelo FK 119

Tabela 11 - Resultados da estimação com o painel C pelo FK 121

Tabela 12 - Resultados da estimação com o painel E pelo FK 122

Tabela 13 - Resultados da estimação com o painel A pelo FP 123

Tabela 14 - Resultados da estimação com o painel D pelo FP 123

Tabela 15 - Resultados da estimação com o painel C pelo FP 126

Tabela 16 - Resultados da estimação com o painel E pelo FP 127

Tabela 17 - Efeito da eficiência do FP na verossimilhança 127

Tabela 18 - Erros entre as variáveis filtrada e observada para o FK e o FP 132

Tabela 19 - Erros entre as variáveis observada e filtrada para o FK e FP para o painel C 134

Tabela 20 - Erros de previsão fora da amostra para o FK e o FP de 12/08/04 a $30 / 12 / 04$

Tabela 21 - Erros de previsão fora da amostra para o FK e o FP de 21/09/00 a $12 / 12 / 01$

Tabela 22 - Resultados da estimação com o painel A pelo FK

Tabela 23 - Resultados da estimação com o painel C pelo FK

Tabela 24 - Resultados da estimação com o painel E pelo FK 
Tabela 25 - Resultado da estimação com o painel A pelo FP

Tabela 26 - Resultados da estimação com o painel C pelo FP

Tabela 27 - Resultados da estimação com o painel E pelo FP

Tabela 28 - Resultados da estimação com o painel A

Tabela 29 - Resultado da estimação com o painel C

Tabela 30 - Estrutura a termo dos preços resultante da estimação com o painel A

Tabela 31 - Estrutura a termo dos preços resultante da estimação com o painel C

Tabela 32 - Estrutura a termo dos preços para o algoritmo de alisamento do painel A

Tabela 33 - Resultados da estimação com o painel A

Tabela 34 - Resultados da estimação com o painel C

Tabela 35 - Estrutura a termo dos preços resultante da estimação com o painel A

Tabela 36 - Estrutura a termo dos preços resultante da estimação com o painel C

Tabela 37 - Resultados da estimação com o painel A 


\section{Lista de quadros}

Quadro 1 - Esquema mostrando os níveis de preços até o vencimento

Quadro 2 - Exemplo com preços em contango e situação de normal backwardation

Quadro 3 - Representação esquemática do filtro de Kalman 79

Quadro 4 - Representação esquemática do filtro de Kalman estendido 84

Quadro 5 - Algoritmo do filtro de partículas 94 


\section{Lista de símbolos e abreviações}

\begin{tabular}{|c|c|}
\hline $\mathrm{bbl}(\mathrm{s})$ & Volume em barril ou barris \\
\hline BDS & Teste de independência de Brock, Dechert e Sheinkman \\
\hline $\mathrm{BM} \& \mathrm{~F}$ & Bolsa Mercantil e de Futuros \\
\hline CBOT & Chigaco Board of Trade \\
\hline CME & Chigaco Mercantile Exchange \\
\hline $\mathrm{E}^{\mathrm{Q}}(\mathrm{Y})$ & Valor esperado de Y com relação à medida de prob. Q \\
\hline EDO & Equação diferencial ordinária \\
\hline EDP & Equação diferencial parcial \\
\hline $\mathfrak{I}$ & $\sigma$-álgebra \\
\hline FAC & Função de autocorrelação \\
\hline $\mathrm{FACP}$ & Função de autocorrelação parcial \\
\hline FK & Filtro de Kalman \\
\hline FKE & Filtro de Kalman estendido \\
\hline FP & Filtro de partículas \\
\hline FPB & Filtro de partículas bootstrap \\
\hline $\mathrm{F}(\mathrm{x})$ & Contrato futuro com vencimento $\mathrm{x}$ meses à frente \\
\hline GMM & Método generalizado dos momentos \\
\hline iid & Independente e identicamente distribuída \\
\hline $\ln$ & Logaritmo na base e \\
\hline MAE & Erro absoluto médio \\
\hline MAPE & Erro absoluto percentual médio \\
\hline $\mathrm{MC}$ & Monte Carlo \\
\hline $\mathrm{MCCM}$ & Monte Carlo cadeia de Markov \\
\hline MGB & Movimento geométrico Browniano \\
\hline MME & Medida martingal equivalente \\
\hline $\mathrm{N}\left(\mu, \sigma^{2}\right)$ & Distribuição normal com média $\mu$ e variância $\sigma^{2}$ \\
\hline NYMEX & New York Mercantile Exchange \\
\hline$\stackrel{\mathrm{qc}}{\rightarrow}$ & $\mathrm{A}_{\mathrm{n}} \stackrel{\mathrm{qc}}{\rightarrow} \mathrm{A}:$ convergência quase-certamente \\
\hline$\Omega$ & Espaço amostral \\
\hline $\mathrm{P}$ & Medida de probabilidade verdadeira \\
\hline Q & Medida Martingal Equivalente \\
\hline
\end{tabular}




$\begin{array}{ll}\mathfrak{R} & \text { Conjunto dos reais } \\ \mathfrak{R}^{\mathrm{n}} & \text { Espaço Euclidiano n dimensional } \\ \text { RMSE } & \text { Raíz quadrada do erro quadrado médio } \\ \text { S\&P500 } & \text { Índice Standart \& Poors } 500 \\ \Theta & \text { Conjunto de hiperparâmetros } \\ \text { US\$/bb1 } & \text { Preço do petróleo em dólares americanos por barril } \\ \mathrm{W}_{\mathrm{t}} & \text { Processo padrão de Wiener } \\ \mathrm{WTI} & \text { West Texas Intermediate } \\ \mathrm{x} & \text { Variável de estado } \\ \mathrm{x}^{\prime} & \text { Transposição de x } \\ \mathrm{y} & \text { Variável de observação } \\ {[\Omega, \Im, P]} & \text { Espaço de probabilidade }\end{array}$

